\title{
Simulation of Guided Wave Propagation in Isotropic and Composite Structures using LISA
}

\author{
Kalyan S. Nadella ${ }^{1}$ and Carlos E. S. Cesnik ${ }^{2}$ \\ Dept. of Aerospace Engineering, University of Michigan, Ann Arbor, MI 48109, USA
}

\begin{abstract}
This paper presents a local interaction simulation approach (LISA) numerical method to examine the guided wave propagation in plate and sandwich structures. The method is based on recursive iterative equations, derived from the elastodynamic equilibrium equations. Derivation of the iterative equations with varying spatial discretizations is presented for a generalized orthotropic medium in non-principle axis frame. The new iterative equations have the capability to model generic laminated composite plates and sandwich composite structures. The results address some of the propagation aspects in isotropic plates, laminated composite plates, and simple composite foam core sandwich.
\end{abstract}

\section{Introduction}

Camp OMPOSITE structures have become an integral part of the aviation industry because of their significant advantages such as high specific strength and stiffness as well as long fatigue life over metallic ones. Layered composites are generally used as laminates or sandwich structures. Due to their current and increasing demand, development of an appropriate structural health monitoring (SHM) system is essential to ensure the proper functionality of the structure. Acoustic waves such as Rayleigh and Lamb waves have been used for damage detection in metallic and composite structures. ${ }^{1,2}$ Rayleigh waves propagate on the surface of semi-infinite media, which is a suitable feature in detecting surface and subsurface defects. On the other hand, Lamb waves can travel long distances over the surface as well as through the thickness of a structure. Therefore, guided waves (GW) based on Lamb waves present promising possibilities in developing SHM systems. By controlling the testing parameters, GW can be sensitive to specific defects in terms of both location and size of the damage. In addition to damage detection, GW are capable of providing the overall degradation state of the material in terms of stiffness change. Studies $^{3}$ have shown GW as an efficient method for damage detection in metallic structures, which motivates similar research in the field of composite materials. Wave propagation has been studied extensively for isotropic materials, but studies for composite structures are still in its dawn. A good understanding of the GW propagation is required to build robust and reliable SHM systems.

The literature describes many methods used by researchers to study wave propagation due to surface point loads, such as 3D elasticity-based theories and approximate plate theories, ${ }^{4}$ and finite-element normal mode expansion formulations. ${ }^{5}$ Theories were also used to model GW generated by finite-dimension piezoelectric transducers ${ }^{6}$ in composite plates. Semi-analytical matrix methods such as the global matrix approach ${ }^{7}$ and transfer matrix ${ }^{8}$ approaches have been used to model ultrasonic waves which are efficient in modeling waves in infinite media. Although analytical/semi-analytical models are able to predict the GW propagation, they are restricted to simple composite structures. Modeling boundary reflections, damage interaction, and geometric complexities become extremely complicated using analytical models.

Several numerical computational methods have been proposed to address the complications arising from modeling of complex structures. Traditional numerical tools such as finite difference (FD) equations ${ }^{9}$ and the finite element (FE) method ${ }^{10}$ have been used extensively for modeling GW propagation. Derivatives of traditional methods such as the boundary element method (BEM) ${ }^{11}$ have been developed to increase the computational efficiency at the cost of limiting it to infinite media and simple structures. FD equations are derived by using second-order or higher-order approximations for resolving spatial derivatives. FD methods can become computationally taxing when complex boundary conditions are involved.

\footnotetext{
${ }^{1}$ Graduate Student Research Assistant; e-mail:nkalyan@umich.edu. AIAA Student Member

${ }^{2}$ Corresponding author: Professor of Aerospace Engineering; ph: (734)764-3397; e-mail: cesnik@umich.edu. Fellow, AIAA.
} 
Recently, it has been shown that the local interaction simulation approach (LISA), ${ }^{12,13}$ a numerical method based on finite difference (FD) transformations, is capable of efficiently and accurately modeling GW propagation. This method is based on iterative equations (IE) for "unit cells" that are used to represent/discretize the model. The actual IEs are derived from the elastodynamic equilibrium equations. LISA was developed to study wave propagation in 3D isotropic heterogeneous media. ${ }^{12}$ LISA's efficiency lies in replacing the second-order spatial and temporal derivatives in the elastodynamic equilibrium equations by recursive relations based on FD transformations. LISA employs the sharp interface model (SIM) to address the issue of discontinuities and boundaries, which distinguishes it from the formal FD techniques, which use smoothing parameters. LISA has been used in the past to study the wave propagation characteristics ${ }^{15,17}$ and accuracy of simulations ${ }^{20}$ in isotropic plates. Moreover, damage interaction and identification ${ }^{16}$ was addressed in isotropic plates. It was further used to study isotropic structures with more complex geometries ${ }^{18}$ and wave propagation through the thickness of a constant orthotropic layer plate structure. ${ }^{19}$ The orthotropic plates were comprised of single layer ${ }^{14}$ or plies having the same properties and were discretized with uniform cell sizes. ${ }^{18}$ The implementation was modified for layered composite laminates ${ }^{20}$ along with variable spatial discretizations.

The overall goal of the present work is to model wave propagation in complex composite structures such as sandwich panels and reinforced composite panels. In this paper, LISA iterative equations are derived for varying spatial discretizations, which will enable the modeling of sandwich composite structures where the facesheet and core need to be discretized differently. Frequency variation studies are carried out on isotropic and orthotropic structures to validate the LISA capability to model various testing parameters. Preliminary experimental and numerical studies are also performed on composite foam core sandwich structures.

\section{Theoretical Formulation}

The theoretical formulation starts with the elastodynamic equations, which are notationally simplified with the help of Voigt's notation. FD approximations are then applied to the modified equations to obtain iterative equations for a homogeneous medium. SIM is employed at the interface of different materials to derive the necessary recursive relations. Finally, the displacement components at a particular node can be written as a function of displacements at previous two time steps of the neighboring nodes. Readers are referred to Refs. 14 and 21 for details pertaining to the basic LISA derivation. The outline for the derivation is shown in Fig. 1, where the dark colored boxes represent the modification to the existing theory. ${ }^{12}$

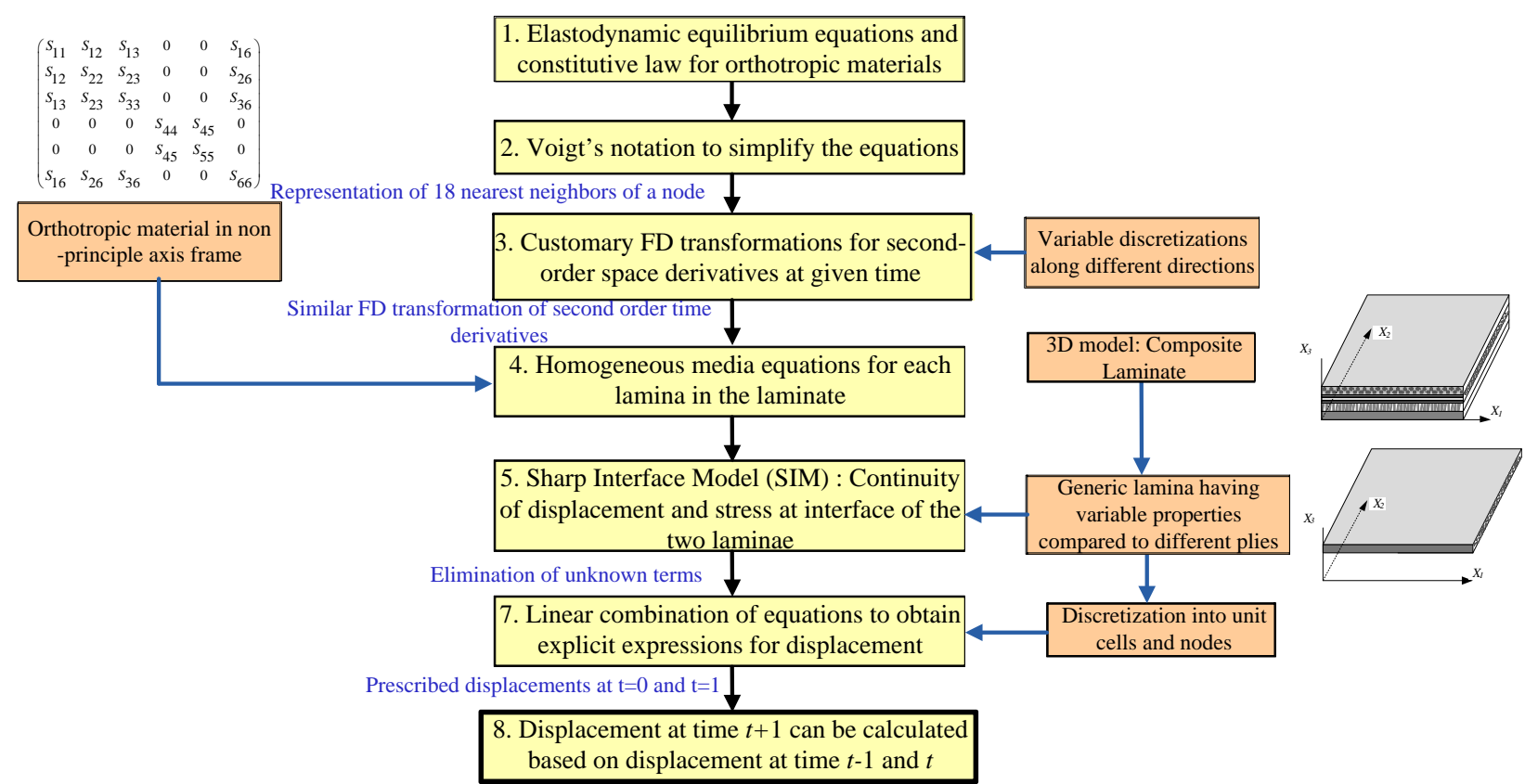

Figure 1. Overview of LISA's theoretical formulation. 


\section{A. Equations of motion}

Consider the equilibrium equations for an orthotropic material, expressed in displacement form as:

$$
\partial_{l}\left(\boldsymbol{S}_{k l m n} w_{m, n}\right)=\rho \ddot{w}_{k} \quad(k, l, m, n=1,2,3)
$$

where $S$ is the stiffness tensor, $\rho$ the material density, and $w$ the displacement field. The subscripts followed by a comma denote spatial differentiation and dot represents differentiation with respect to time. Voigt's notation is used, which converts two indices into a single index, and simplifies the notation for the analysis, i.e.,

$$
\begin{array}{lr}
(n, l) \rightarrow \zeta=n \delta_{n l}+\left(1-\delta_{n l}\right)(9-n-l) & (\zeta=1, . ., 6) \\
(k, m) \rightarrow \eta=k \delta_{k m}+\left(1-\delta_{k m}\right)(9-k-m) & (\eta=1, . ., 6)
\end{array}
$$

resulting in

$$
\sum_{m=1}^{3} \sum_{\eta=1}^{6} \boldsymbol{S}_{\eta \zeta} w_{m, \zeta}=\rho \ddot{\mathbf{w}}_{k}
$$

where the indices $k, m, n$ and $l$ assume values 1,2 and 3, and $\delta$ is the Dirac-delta function. Previously the method was derived for orthotropic plates oriented along the principle axis, in which most of the off-diagonal terms are zero. For most of the practical applications, the orthotropic materials are used with different lay-up angles to construct the required composite structure. To address this issue, in-plane rotation of the orthotropic medium is considered in the formulation. The new transformed stiffness matrix ${ }^{23} S$ with in-plane rotation becomes:

$$
S=\left(\begin{array}{cccccc}
S_{11} & S_{12} & S_{13} & 0 & 0 & S_{16} \\
S_{12} & S_{22} & S_{23} & 0 & 0 & S_{26} \\
S_{13} & S_{23} & S_{33} & 0 & 0 & S_{36} \\
0 & 0 & 0 & S_{44} & S_{45} & 0 \\
0 & 0 & 0 & S_{45} & S_{55} & 0 \\
S_{16} & S_{26} & S_{36} & 0 & 0 & S_{66}
\end{array}\right)
$$

\section{B. Discretization and FD transformations}

All 18 neighboring points around a given point $C$ (as shown in Fig. 2(a)) are required to account for the double spatial derivatives. The finite difference expressions used in the derivation are shown for only $W$ ( $w_{3}$-component), as the other components of displacement $U$ ( $w_{1}$-component) and $V$ ( $w_{2}$-component) have similar expressions. For convenience, the subscripts as well as the commas in the subscripts are dropped. The basic finite difference expressions for the derivation can be found in Sinor, ${ }^{21}$ as the expressions shown in this document emphasize the modifications to the original ones. A grid with non-uniform spatial steps is considered for the derivation. Finite difference relations are sufficient to transform the double spatial derivatives in space into recursive relations for homogenous materials. For heterogeneous structures, the $\mathrm{SIM}^{12}$ is used to calculate the response at the "crosspoints" (the lattice points at the intersection of cells). The SIM assumes that the stress and displacement variables are uniform within a cell, and to maintain equilibrium and continuity, they are matched at the interface between cells. The extra conditions provided by the SIM incorporate the changes in stiffness, density, or attenuation properties into the iterative equations. 


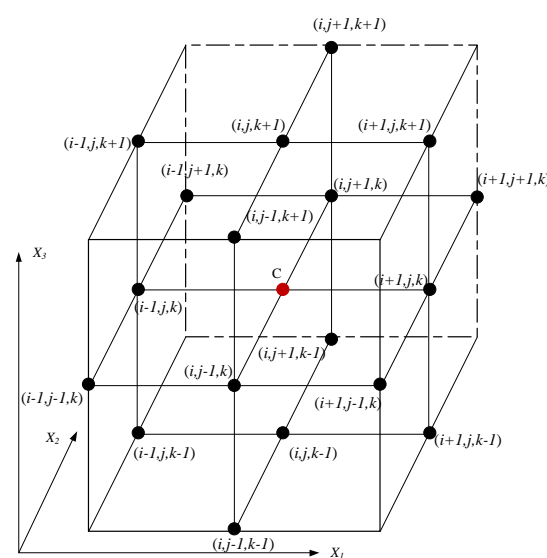

(a)

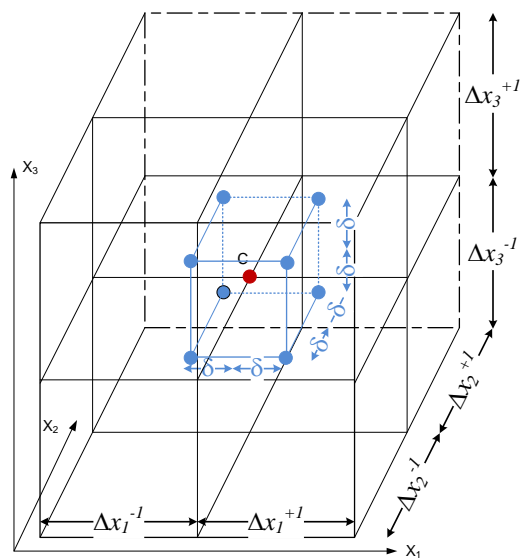

(b)

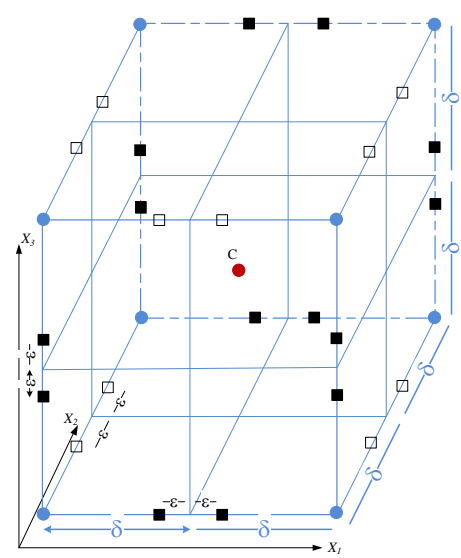

(c)

Figure2. (a) A generic point $C(i, j, k)$ with 18 nearest neighbors and the Cartesian system used in the analysis. (b) Blue dots show the 8 points chosen to enforce displacement continuity. (c) Additional points shown as squares are used to enforce stress continuity conditions.

\section{Displacement and stress continuity}

Eight additional points are considered as shown in Fig. 2(b) at a distance of $\delta<\Delta x_{i}$ (i=1,2,3), given by $(i+\alpha \delta, j+\beta \delta, k+\gamma \delta)$ for $\alpha, \beta, \gamma= \pm 1$. Elastodynamic equilibrium equations shown in Eq. (3) are represented at these additional points as:

$$
\sum_{m=1}^{3} \sum_{\eta=1}^{6} S_{\eta \zeta}^{i+\alpha \delta, j+\beta \delta, k+\gamma \delta} w_{m, \zeta}^{i+\alpha \delta, j+\beta \delta, k+\gamma \delta}=\rho \ddot{w}_{k}^{i+\alpha \delta, j+\beta \delta, k+\gamma \delta} \quad \alpha, \beta, \gamma= \pm 1, k=1,2,3
$$

The resulting equations for a single component of displacement, say $W\left(w_{3}\right)$, can be combined at these new points while $\ddot{W}$ is enforced to be the same at the eight points to obtain the continuity of displacements for $W$. Similarly, continuity for $U\left(w_{1}\right)$ and $V\left(w_{2}\right)$ can be imposed. The second order derivatives in Eq. (5) are resolved as:

$$
\begin{aligned}
W_{x_{1} x_{1}}^{i+\alpha \delta, j+\beta \delta, k+\gamma \delta} & =\frac{W_{x_{1}}^{i+\alpha / 2, j, k}-W_{x_{1}}^{i+\alpha, j+\beta, k+\gamma}}{\alpha \cdot \Delta x_{1}^{\alpha}} \\
W_{x_{1} x_{2}}^{i+\alpha \delta, j+\beta \delta, k+\gamma \delta} & =\frac{W^{i+\alpha, j+\beta, k}-W^{i+\alpha, j, k}-W^{i, j+\beta, k}+W^{i, j, k}}{\alpha \cdot \beta \cdot \Delta x_{1}^{\alpha} \cdot \Delta x_{2}^{\beta}} \\
W_{x_{3} x_{1}}^{i+\alpha \delta, j+\beta \delta, k+\gamma \delta} & =\frac{W^{i+\alpha, j, k+\gamma}-W^{i+\alpha, j, k}-W^{i, j, k+\gamma}+W^{i, j, k}}{\alpha \cdot \gamma \cdot \Delta x_{1}^{\alpha} \cdot \Delta x_{3}^{\gamma}}
\end{aligned}
$$

where the remaining spatial derivatives have similar form. The first order derivatives in Eq. (6) are resolved as:

$$
\begin{aligned}
W_{x_{1}}^{i+\alpha / 2, j, k} & =\frac{W^{i+\alpha, j, k}-W^{i, j, k}}{\alpha \cdot \Delta x_{1}^{\alpha}} \\
W_{x_{2}}^{i, j+\beta / 2, k} & =\frac{W^{i, j+\beta, k}-W^{i, j, k}}{\beta \cdot \Delta x_{2}^{\beta}} \\
W_{x_{3}}^{i, j, k+\gamma / 2} & =\frac{W^{i, j, k+\gamma}-W^{i, j, k}}{\gamma \cdot \Delta x_{3}^{\gamma}}
\end{aligned}
$$

where the superscript for $W$ denotes a particular node in Fig. 2(a). It should be noted that first derivative of $W^{i+\alpha \delta, j+\beta \delta, k+\gamma \delta}$ for $\alpha, \beta, \gamma= \pm 1$ remains unevaluated and is eliminated while the stress continuity is enforced. To obtain 
continuity of stress, additional points are introduced $(i+\alpha \varepsilon, j+\beta \delta, k+\gamma \delta),(i+\alpha \delta, j+\beta \varepsilon, k+\gamma \delta)$ and $(i+\alpha \delta, j+\beta \delta, k+\gamma \varepsilon)$ with $\varepsilon<<\delta<\Delta x_{i}(i=1,2,3)$ represented as black squares in Fig. 2(c). The derivatives involving $\varepsilon$ are resolved as:

$$
\begin{aligned}
W_{x_{1}}^{i+\alpha \varepsilon, j+\beta \delta, k+\gamma \delta} & =W_{x_{1}}^{i+\alpha \delta, j+\beta \delta, k+\gamma \delta} \\
W_{x_{1}}^{i+\alpha \delta, j+\beta \varepsilon, k+\gamma \delta} & =\frac{W^{i+\alpha, j, k}-W^{i, j, k}}{\alpha \cdot \Delta x_{1}^{\alpha}} \\
W_{x_{2}}^{i+\alpha \delta, j+\beta \delta, k+\gamma \varepsilon} & =\frac{W^{i, j+\beta, k}-W^{i, j, k}}{\beta \cdot \Delta x_{2}^{\beta}}
\end{aligned}
$$

Similar expressions follow for the other terms. It should be noted that the first derivative terms at the eight points shown in Fig. 2(b) remain unevaluated and are eliminated based on the stress continuity relations. The stress components $\left(\tau_{k l}\right)$ can be written as:

$$
\tau_{k l}=\boldsymbol{S}_{k l m n} w_{m, x_{n}}
$$

Next, the stress continuity is enforced with the help of the following relations:

$$
\begin{aligned}
& \tau_{k 1}^{i+\varepsilon, j+\beta \delta, k+\gamma \delta}=\tau_{k 1}^{i-\varepsilon, j+\beta \delta, k+\gamma \delta} \\
& \tau_{k 2}^{i+\alpha \delta, j+\varepsilon, k+\gamma \delta}=\tau_{k 2}^{i+\alpha \delta, j-\varepsilon, k+\gamma \delta} \\
& \tau_{k 3}^{i+\alpha \delta, j+\beta \delta, k+\varepsilon}=\tau_{k 3}^{i+\alpha \delta, j+\beta \delta, k-\varepsilon}
\end{aligned} \quad(k=1,2,3)
$$

Using Eq. (5) at the eight points $(i+\alpha \delta, j+\beta \delta, k+\gamma \delta)$ and the select group of stress continuity relations given in Eq. (10), the final iterative relations for the three-displacement components are derived for the 3D case as:

$$
\begin{aligned}
& U^{i, j, k, t+1}=-U^{i, j, k, t-1}+2 U^{i, j, k}+\frac{2 \chi}{8} U^{i, j, k} \underset{\alpha, \beta, \gamma= \pm 1}{\sum}\left[\left(\eta_{x}^{2} \tilde{s}_{11}+\eta_{y}^{2} \tilde{s}_{12}+\eta_{z}^{2} \tilde{S}_{13}\right)\right] \\
& +\frac{\chi}{8} \underset{\alpha, \beta, \gamma= \pm 1}{\sum_{1}}\left[2 \eta_{x}^{2} \tilde{s}_{11} U^{i+\alpha, j, k}+2 \eta_{y}^{2} \tilde{s}_{12} U^{i, j+\beta, k}+2 \eta_{z}^{2} \tilde{s}_{13} U^{i, j, k+\gamma}\right] \\
& +\frac{\chi}{8} \underset{\alpha, \beta, \gamma= \pm 1}{\sum_{1}}\left[\alpha \beta \eta_{x} \eta_{y}\left\{\left(\tilde{s}_{12}+\tilde{s}_{66}\right)\left(v^{i+\alpha, j+\beta, k}-V^{i, j, k}\right)+\left(\tilde{s}_{12}-\tilde{s}_{66}\right)\left(v^{i, j+\beta, k}-V^{i+\alpha, j, k}\right)\right\}\right] \\
& +\frac{\chi}{8} \underset{\alpha, \beta, \gamma= \pm 1}{\sum^{2}}\left[\alpha \gamma \eta_{\chi} \eta_{z}\left\{\left(\tilde{S}_{13}+\tilde{S}_{55}\right)\left(w^{i+\alpha, j, k+\gamma}-w^{i, j, k}\right)+\left(\tilde{S}_{13}-\tilde{S}_{55}\right)\left(w^{i, j, k+\gamma}-w^{i+\alpha, j, k}\right)\right\}\right]
\end{aligned}
$$

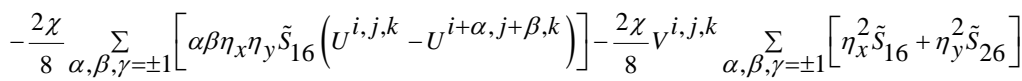

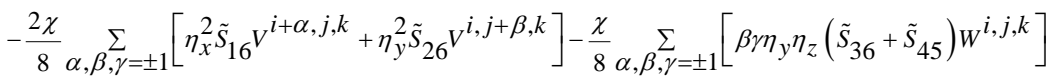

$$
\begin{aligned}
& +\frac{\chi}{8} \sum_{\alpha, \beta, \gamma= \pm 1}\left[\beta \gamma \eta_{y} \eta_{z} \tilde{S}_{36}\left(w^{i, j+\beta, k+\gamma}+w^{i, j, k+\gamma}-w^{i, j+\beta, k}\right)\right] \\
& +\frac{\chi}{8} \sum_{\alpha, \beta, \gamma= \pm 1}\left[\beta \gamma \eta_{y} \eta_{z} \tilde{s}_{45}\left(w^{i, j+\beta, k+\gamma}-w^{i, j, k+\gamma}+w^{i, j+\beta, k}\right)\right]+\frac{2 \chi}{8} \sum_{\alpha, \beta, \gamma= \pm 1} \eta_{z}^{2} \tilde{s}_{45}\left(V^{i, j, k+\gamma}-V^{i, j, k}\right)
\end{aligned}
$$




$$
\begin{aligned}
& V^{i, j, k, t+1}=-V^{i, j, k, t-1}+2 V^{i, j, k}-\frac{2 \chi}{8} V^{i, j, k} \sum_{\alpha, \beta, \gamma= \pm 1}\left[\eta_{x}^{2} \tilde{s}_{12}+\eta_{y}^{2} \tilde{s}_{22}+\eta_{z}^{2} \tilde{s}_{23}\right] \\
& +\frac{\chi}{8} \underset{\alpha, \beta, \gamma= \pm 1}{ }\left[2 \eta_{x}^{2} \tilde{s}_{12} V^{i+\alpha, j, k}+2 \eta_{y}^{2} \tilde{s}_{22} V^{i, j+\beta, k}+2 \eta_{z}^{2} \tilde{s}_{23} V^{i, j, k+\gamma}\right] \\
& +\frac{\chi}{8} \underset{\alpha, \beta, \gamma= \pm 1}{\sum}\left[\alpha \beta \eta_{x} \eta_{y}\left\{\left(\tilde{S}_{12}+\tilde{S}_{66}\right)\left(U^{i+\alpha, j+\beta, k}-U^{i, j, k}\right)+\left(\tilde{S}_{12}-\tilde{S}_{66}\right)\left(U^{i+\alpha, j, k}-U^{i, j+\beta, k}\right)\right\}\right] \\
& +\frac{\chi}{8} \underset{\alpha, \beta, \gamma= \pm 1}{\sum_{1}}\left[\beta \gamma \eta_{y} \eta_{z}\left\{\left(\tilde{S}_{23}+\tilde{S}_{44}\right)\left(w^{i, j+\beta, k+\gamma}-w^{i, j, k}\right)+\left(\tilde{S}_{23}-\tilde{S}_{44}\right)\left(w^{i, j, k+\gamma}-w^{i, j+\beta, k}\right)\right\}\right] \\
& -\frac{2 \chi}{8} \sum_{\alpha, \beta, \gamma= \pm 1}\left[\alpha \beta \eta_{x} \eta_{y} \tilde{S}_{26}\left(V^{i, j, k}-V^{i+\alpha, j+\beta, k}\right)\right]-\frac{2 \chi}{8} U^{i, j, k} \sum_{\alpha, \beta, \gamma= \pm 1}\left[\eta_{x}^{2} \tilde{S}_{16}+\eta_{y}^{2} \tilde{S}_{26}\right] \\
& -\frac{2 \chi}{8} \sum_{\alpha, \beta, \gamma= \pm 1}\left[\eta_{\chi}^{2} \tilde{s}_{16} U^{i+\alpha, j, k}+\eta_{y}^{2} \tilde{s}_{26} U^{i, j+\beta, k}\right]-\frac{\chi}{8} \sum_{\alpha, \beta, \gamma= \pm 1}\left[\alpha \gamma \eta_{\chi} \eta_{z}\left(\tilde{s}_{36}+\tilde{S}_{45}\right) w^{i, j, k}\right] \\
& +\frac{\chi}{8} \sum_{\alpha, \beta, \gamma= \pm 1}\left[\alpha \gamma \eta_{x} \eta_{z} \tilde{S}_{36}\left(w^{i, j+\beta, k+\gamma}+W^{i, j, k+\gamma}-W^{i+\alpha, j, k}\right)\right] \\
& +\frac{\chi}{8} \underset{\alpha, \beta, \gamma= \pm 1}{\sum}\left[a \gamma \eta_{x} \eta_{Z} \tilde{s}_{45}\left(w^{i, j+\beta, k+\gamma}-w^{i, j, k+\gamma}+w^{i+\alpha, j, k}\right)\right]+\frac{2 \chi}{8} \underset{\alpha, \beta, \gamma= \pm 1}{\sum_{Z}}\left[\eta_{Z}^{2} \tilde{s}_{45}\left(U^{i, j, k+\gamma}-U^{i, j, k}\right)\right] \\
& W^{i, j, k, t+1}=-W^{i, j, k, t-1}+2 W^{i, j, k}-\frac{2 \chi}{8} W^{i, j, k} \sum_{\alpha, \beta, \gamma= \pm 1}\left[\eta_{x}^{2} \tilde{S}_{13}+\eta_{y}^{2} \tilde{S}_{23}+\eta_{z}^{2} \tilde{s}_{33}\right] \\
& +\frac{\chi}{8} \underset{\alpha, \beta, \gamma= \pm 1}{\sum_{1}}\left[2 \eta_{x}^{2} \tilde{S}_{13} W^{i+\alpha, j, k}+2 \eta_{y}^{2} \tilde{s}_{23} W^{i, j+\beta, k}+2 \eta_{z}^{2} \tilde{s}_{33} W^{i, j, k+\gamma}\right] \\
& +\frac{\chi}{8} \sum_{\alpha, \beta, \gamma= \pm 1}\left[\beta \gamma \eta_{y} \eta_{z}\left\{\left(\tilde{s}_{23}+\tilde{S}_{44}\right)\left(v^{i, j+\beta, k+\gamma}-V^{i, j, k}\right)+\left(\tilde{S}_{23}-\tilde{s}_{44}\right)\left(v^{i, j+\beta, k}-V^{i, j, k+\gamma}\right)\right\}\right] \\
& +\frac{\chi}{8} \sum_{\alpha, \beta, \gamma= \pm 1}\left[\alpha \gamma \eta_{\chi} \eta_{z}\left\{\left(\tilde{S}_{13}+\tilde{S}_{55}\right)\left(U^{i+\alpha, j, k+\gamma}-U^{i, j, k}\right)+\left(\tilde{S}_{13}-\tilde{S}_{55}\right)\left(U^{i+\alpha, j, k}-U^{i, j, k+\gamma}\right)\right)\right]
\end{aligned}
$$

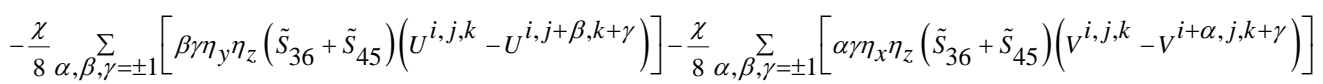

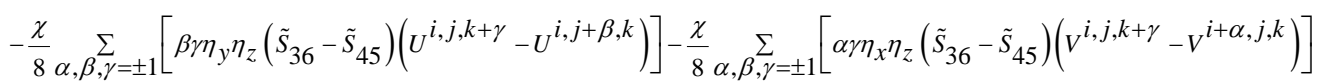

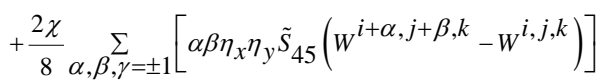

where $U, V$ and $W$ are the components of displacement along the $X_{1}, X_{2}$ and $X_{3}$ axes, respectively. Moreover, $\eta_{x}$ $=1 / \Delta x_{1}{ }^{\alpha}, \eta_{y}=1 / \Delta x_{2}{ }^{\beta}, \eta_{z}=1 / \Delta x_{3}{ }^{\gamma}$, and $\Delta x_{1}{ }^{\alpha}, \Delta x_{2}{ }^{\beta}$, and $\Delta x_{3}{ }^{\gamma}$ are the spatial steps along $X_{1}, X_{2}$ and $X_{3}$ axes, respectively, with $(\alpha, \beta, \gamma)$ equal to \pm 1 . The current time $t$ is assumed where it is not mentioned. "Tilde" over $S_{11}=$ $S_{11}(i+\alpha, j+\beta, k+\gamma)$ represents one of the eight cells surrounding the point $\mathrm{C}$ depending on the choice of $(\alpha, \beta, \gamma)$ from $(+1,-1)$, and similar expressions hold for the other stiffness terms. $\chi=\left(\Delta t^{2} / \rho\right)$, where $\Delta t$ is the time-step used in the simulation and $\rho$ is the average density of all the 8 cells surrounding point $C$ as shown in Fig. 2(a).

The parameters (cuboid grid and time step) used in the discretization of the Eqs. (11)-(13) are selected based on the Courant-Friedric-Lewy (CFL) criterion. The CFL criterion is a constraint that bounds the time step and cell size by the relation:

$$
\mathrm{CFL}=C_{\max } \Delta t \sqrt{\frac{1}{\Delta x_{1}^{2}}+\frac{1}{\Delta x_{2}^{2}}+\frac{1}{\Delta x_{3}^{2}}} \leq 1
$$

where $c_{\max }$ is the maximum wave speed. This criterion ensures proper capture of the wave propagation in time and space for a given time step and grid spacing. The spatial grid sizing should have at least 8 nodes per minimum wavelength, and according to Ref. 22, it is also recommended to have an upper limit of 20 nodes per wavelength to avoid excessively long run times and truncation errors. 


\section{Numerical Studies}

\section{A. Summary of structure test cases}

In this section, the numerical formulation described above is exercised for different structures to validate the capability of the model to capture the GW propagation. In previous studies, ${ }^{20,23}$ the frequency of input excitation was fixed at $75 \mathrm{kHz}$, and cell discretization was uniform through the thickness for the numerical validation. In our current effort, the simulation model is used to model GW propagation at different frequencies and varying cell size through the thickness. The simulations are implemented in Intel \& FORTRAN95. The input excitation for the numerical simulations is a 3.5-cycle Hann-modulated toneburst at varying frequencies, which is modeled on the structure as prescribed in-plane displacements across the span of the actuator. The different material systems used in the simulations are shown in Table 1, and these materials will be subsequently referred to by their material-ID.

Table 1. Material properties used in the simulation

\begin{tabular}{ccccc}
\hline \hline Material Name & Aluminum & $\begin{array}{c}\text { IM7 }- \\
\text { Cycom 977-3 }\end{array}$ & $\begin{array}{c}\text { T300B-3K Fabric }-\begin{array}{c}\text { Last-A-Foam } \\
\text { Epon 862 }\end{array} \\
\text { FR - 6710 }\end{array}$ \\
\hline Material-ID & 1 & 2 & 3 & 4 \\
\hline $\mathrm{E}_{1}(\mathrm{GPa})$ & 70 & 147 & 50.1 & 0.089 \\
$\mathrm{E}_{2}(\mathrm{GPa})$ & 70 & 9.8 & 50.1 & 0.089 \\
$\mathrm{E}_{3}(\mathrm{GPa})$ & 70 & 9.8 & 9.8 & 0.089 \\
\hline$v_{12}$ & 0.33 & 0.405 & 0.21 & 0.3 \\
$v_{23}$ & 0.33 & 0.48 & 0.4 & 0.3 \\
$v_{31}$ & 0.33 & 0.027 & 0.078 & 0.3 \\
\hline $\mathrm{G}_{12}(\mathrm{GPa})$ & 26.32 & 3.7 & 19.6 & 0.0195 \\
$\mathrm{G}_{23}(\mathrm{GPa})$ & 26.32 & 3.31 & 3.5 & 0.0195 \\
$\mathrm{G}_{31}(\mathrm{GPa})$ & 26.32 & 3.7 & 3.5 & 0.0195 \\
\hline$\rho\left(\mathrm{kg} / \mathrm{m}^{3}\right)$ & 2700 & 1558 & 1760 & 160 \\
\hline \hline
\end{tabular}

The 3D structures implemented in the simulation are: a homogeneous plate (material-1), a multilayered unidirectional laminate (material-2), and a sandwich composite panel composed of multi-layer facesheets (material-3) and foam core (material-4). A universal time step of $1 \times 10^{-8} \mathrm{~s}$ is used for all the simulations, and the spatial discretizations are chosen accordingly to ensure numerical stability according to the CFL criterion. To simulate free boundary conditions, additional grid points with the material properties of air (density of $1.3 \mathrm{~kg} / \mathrm{m}^{3}$ and stiffness 10,000 times less than material-1) are added. The out-of-plane displacement component is monitored in the simulation for the top surface of the composite laminate.

\section{B. Results and discussion}

1. Dispersion curve for $A_{0}$ mode

Dispersion curves are generated for a 3-mm thick plate of material-1 and 1.5-mm thick uni-directional laminate made from material-2. The theoretical dispersion curve is obtained by using the DISPERSE ${ }^{26}$ software. The actuation in the numerical model is prescribed with two circular actuators of diameter $13.4 \mathrm{~mm}$ placed on the top and bottom surfaces of the plate as shown in Fig. 3(a). The actuator nodes are excited with an out-of-phase 3.5-cycle Hann-modulated toneburst to simulate the anti-symmetric wave in the plates. 


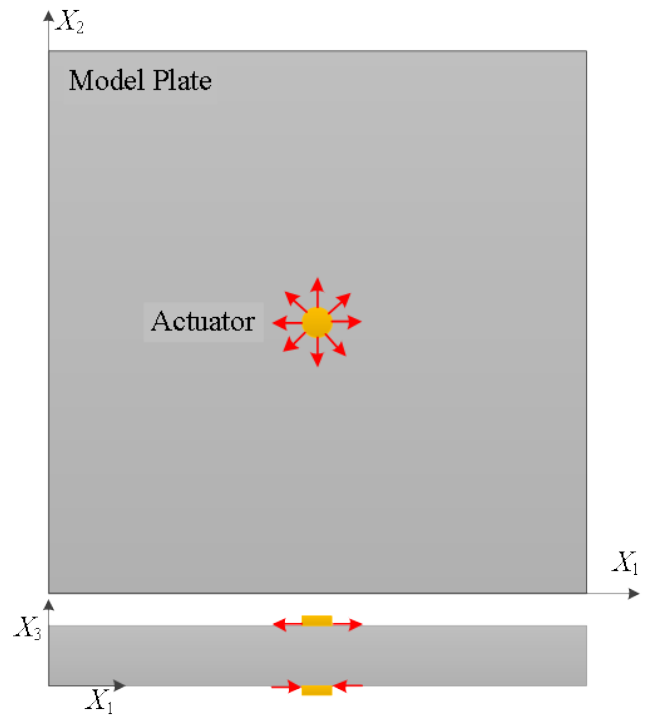

(a)

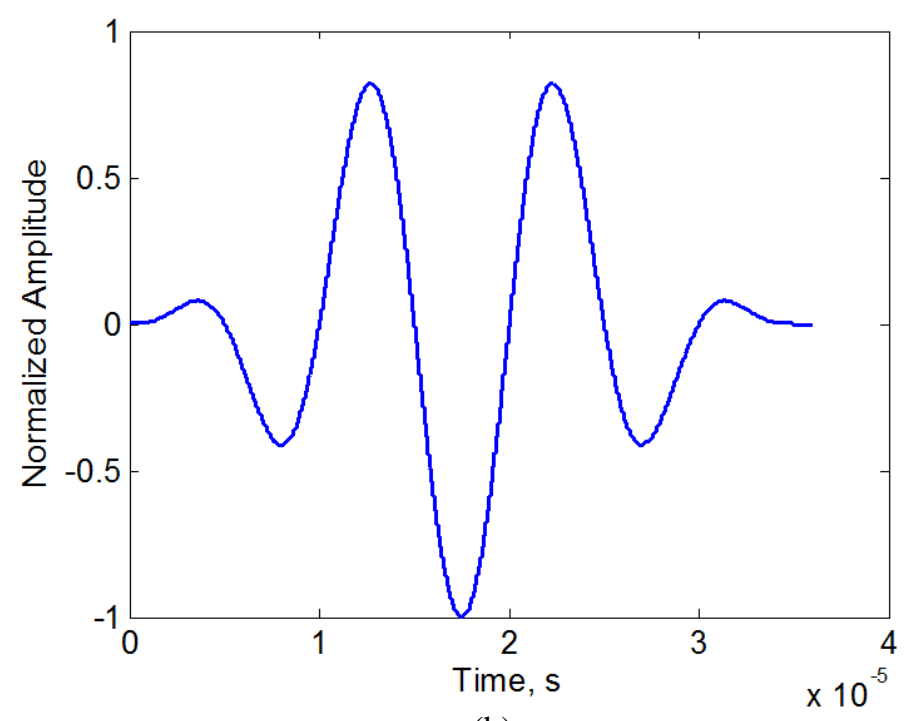

(b)

Figure 3. (a) Experimental setup showing the model plate and actuator placement. (b) Sample excitation based on 3.5 cycle Hann-modulated toneburst.

The out-of-plane displacement $\left(W_{3}\right)$ is monitored on the top surface of the plate/laminate to measure the GW propagation speed. Hilbert transformation is used on the time-history signal to mark the arrival times of the peaks in the signal. The speed of the GW is calculated by measuring the time taken by the signal peak to arrive at nodes which are at distance of 60,70 and $80 \mathrm{~mm}$ from the center of the actuator. The nodes are selected on a radial line from the center of the actuator parallel to the $X_{1}$ axis. The center frequency of the input signal in the LISA simulation for the model plate made of material- 1 is varied from $50 \mathrm{kHz}$ to $250 \mathrm{kHz}$ at an interval of $10 \mathrm{kHz}$, and the group velocity measured is compared with DIPSERSE in Fig. 4(a). For the laminate made from material-2, the center frequency is varied between $50 \mathrm{kHz}$ and $200 \mathrm{kHz}$ at an interval of $10 \mathrm{kHz}$ and the comparison between DISPERSE and LISA can be seen in Fig. 4(b). We can see that there is a good correlation between the speeds predicted by the DISPERSE and LISA simulations. The variation between the speeds calculated from LISA simulations and DISPERSE is under $5 \%$.

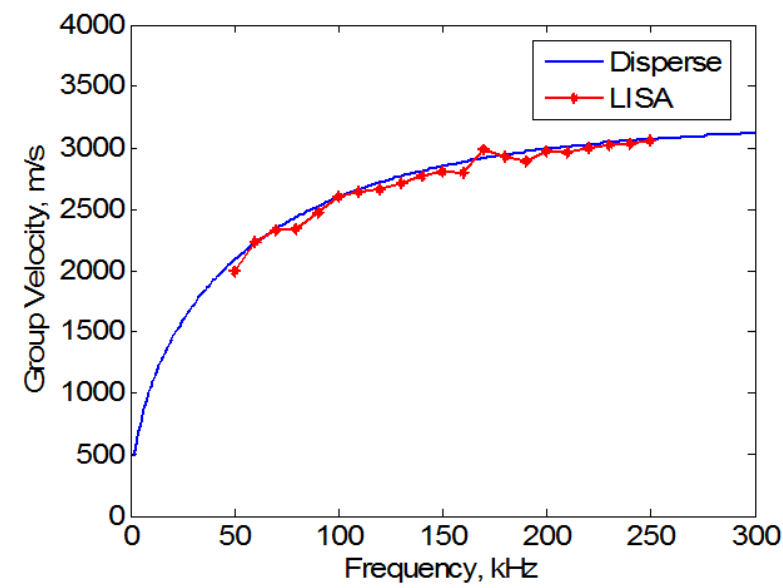

(a)

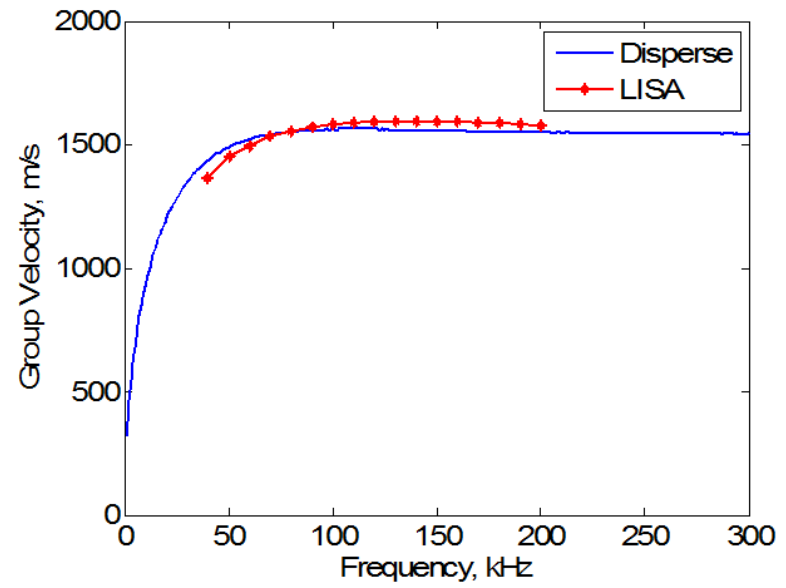

(b)

Figure 4. Comparison between the group velocities for $A_{0}$ mode calculated from LISA and DISPERSE for (a) homogeneous plate (material-1) and (b) IM7 uni-directional laminate (material-2) 


\section{Non-uniform cell discretizations}

Preliminary validation for GW propagation for uni-ply laminates is presented in previous work by the authors. ${ }^{20,23}$ In this study, the new formulation with non-uniform spatial discretizations along the $X_{3}$ axis as shown in Fig. 3 is implemented. A uni-directional laminate made of material-2 is considered for this study. The laminate model is excited with in-plane displacements by a circular actuator of radius $6.4 \mathrm{~mm}$ on the top-surface, shown as an orange dot in Fig. 5, and it is excited with a 3.5-cycle Hann-modulated toneburst with center frequency of $75 \mathrm{kHz}$. In all the results shown in Fig. 5, the fiber direction is coincident with the horizontal direction. The through-thethickness discretization $\left(\Delta x_{3}\right)$ is varied for different simulations as shown in the first column in Fig. 5, and the outof-plane displacements on the top-surface are recorded to verify the capability of the modified iterative equations to incorporate the variation in $\Delta x_{3}$.

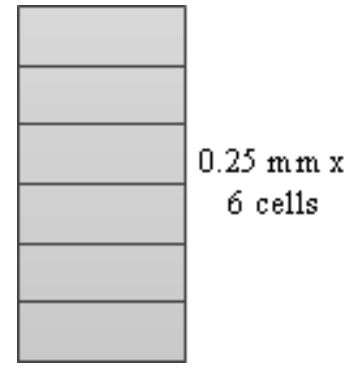

(a)

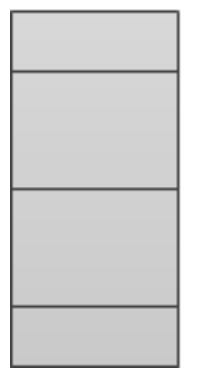

\section{$0.25 \mathrm{~mm} \mathrm{x}$ 1 cell \\ $0.5 \mathrm{~mm} \mathrm{x}$ \\ 2 cells \\ $0.25 \mathrm{~mm} x$ \\ 1 cell}

(e)

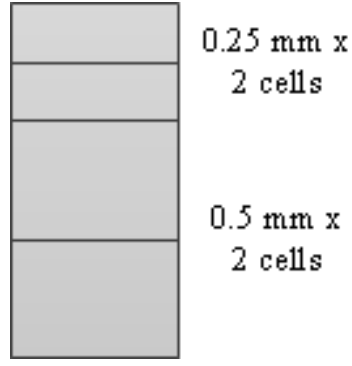

(i)

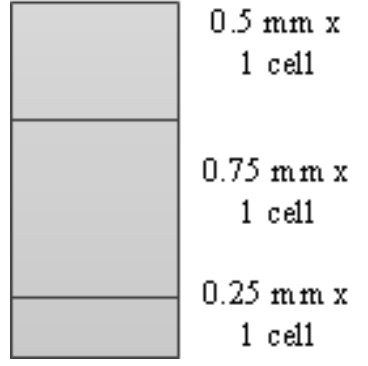

(q)

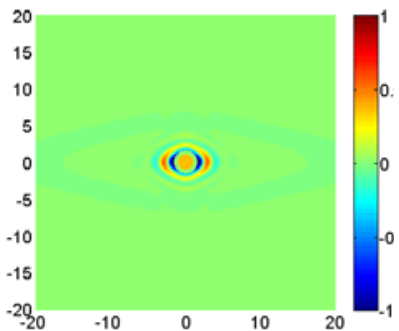

(b) $20 \mu \mathrm{s}$

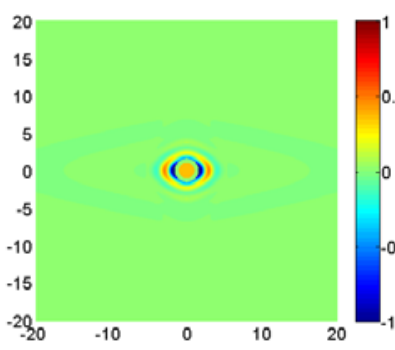

(f) $20 \mu \mathrm{s}$

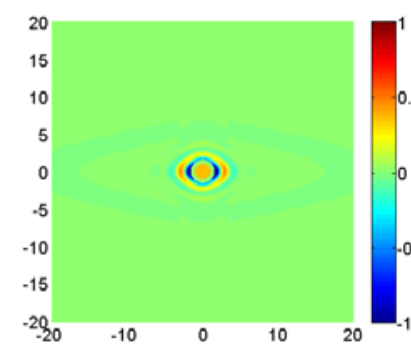

(j) $20 \mu \mathrm{s}$

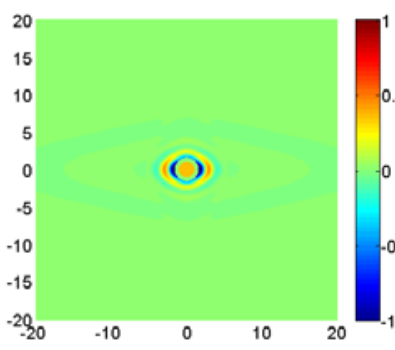

(r) $20 \mu \mathrm{s}$

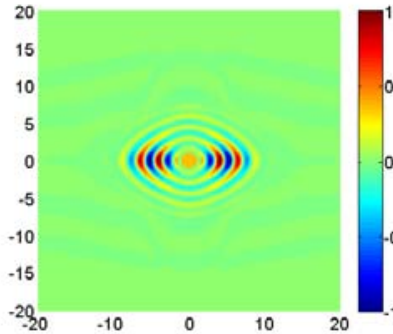

(c) $40 \mu \mathrm{s}$

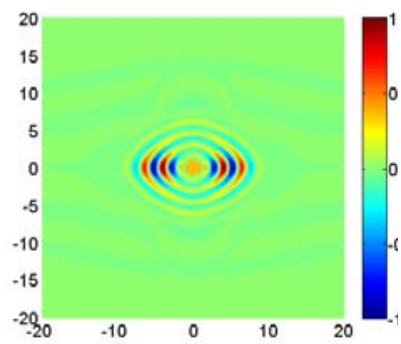

(g) $40 \mu \mathrm{s}$

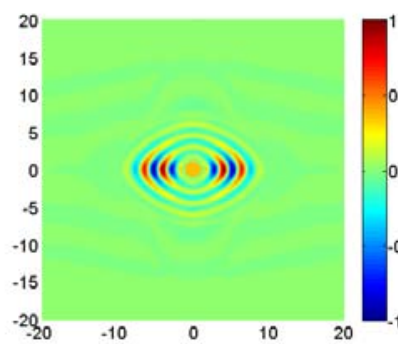

(k) $40 \mu \mathrm{s}$

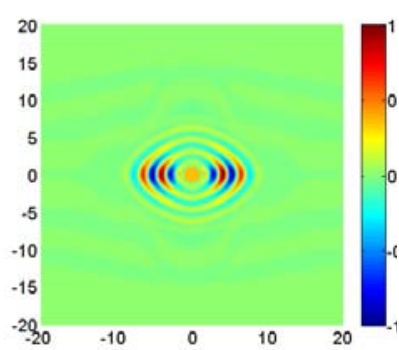

(s) $40 \mu \mathrm{s}$

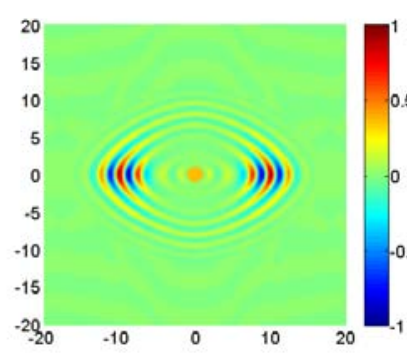

(d) $60 \mu \mathrm{s}$

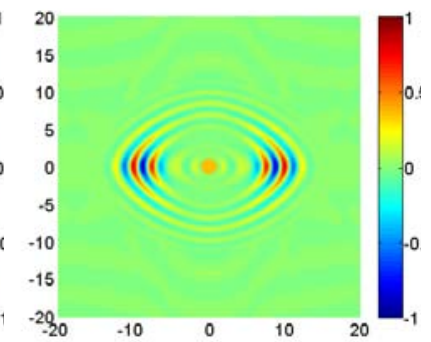

(h) $60 \mu \mathrm{s}$

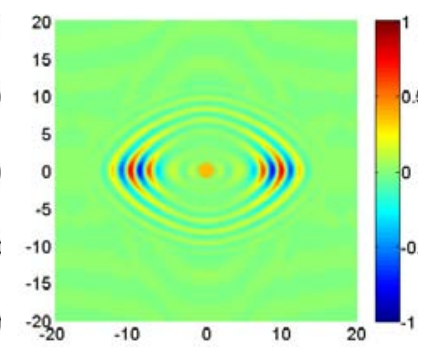

(l) $60 \mu \mathrm{s}$

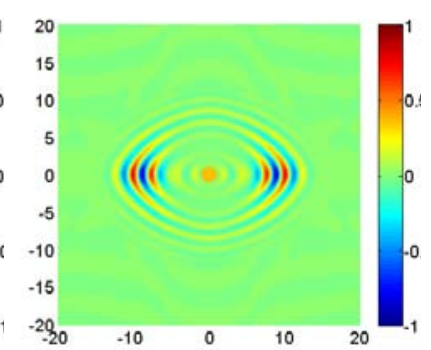

(t) $60 \mu \mathrm{s}$

Figure 5. Out-of-plane displacement pattern for uni-ply laminate when $\Delta x_{3}$ is varied. Through-the-thickness discretization is shown in col. 1 and the snapshots of the propagating waveform are shown in successive columns. 
In Fig. 5, the $X_{1}$ and $X_{2}$ dimensions of the plate are normalized by the radius of the actuator. The plots shown in Figs. 5(a)-(d) are the baseline results calculated using the uniform thickness discretizations. Figures. 5(e), 5(i), and 5(q) show the different discretizations used in this study where the cell size and number of cells through the thickness are varied, but the total thickness is kept constant at $1.5 \mathrm{~mm}$. From the plots that follow a particular discretization in the first column of Fig. 5, one can see that the new iterative equations are able to capture the GW propagation accurately with the non-uniform through-the-thickness discretization.

\section{Preliminary $G W$ studies in sandwich foam core panels}

Preliminary experimental and numerical studies using LISA are performed to assess the feasibility of GW approaches for delamination monitoring of the interface between the sandwich core and the composite facesheets. The particular architecture of interest, shown in Fig. 6(a), consists of surface-bonded transducers bonded on the inner part of an aerospace structure to emit GW used for interrogation of the interface between the sandwich core and the outer composite facesheet.

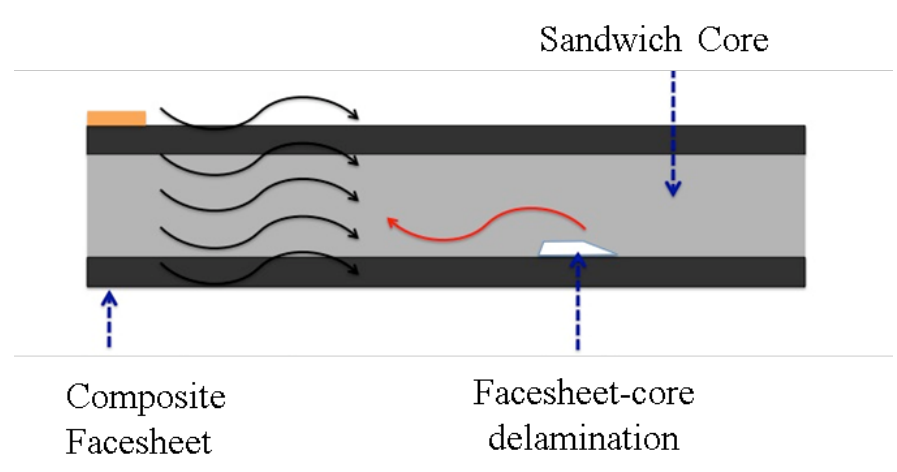

(a)

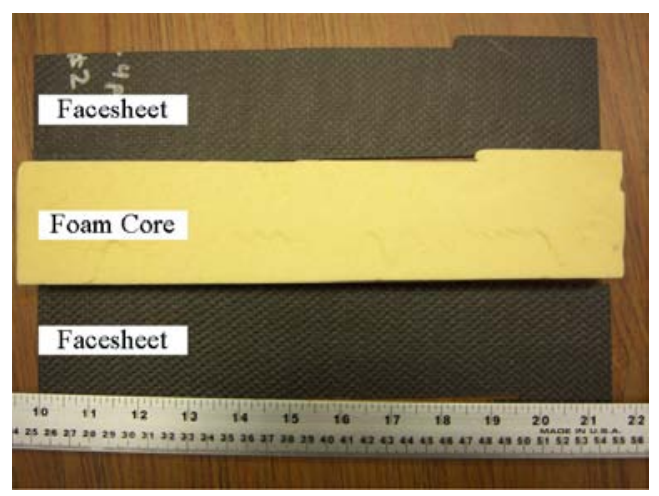

(b)

Figure 6. (a) Schematic of GW-based damage detection architecture for sandwich panels. (b) Components of the sandwich foam core.

The composite sandwich specimen composed of a closed-cell polyurethane foam LAST-A-FOAM ${ }^{\circledR}$ FR-6710 (material-4) sandwiched between facesheets comprising of four plies of T300B-3K (material-3) plain weave carbon fiber fabric with the Epon 862 epoxy is shown in Fig. 6(b). The facesheets and core have thickness of $1 \mathrm{~mm}$ and 24 $\mathrm{mm}$, respectively. The mechanical properties are obtained from Refs. 24 and 25 . The specimen had a $0.5 \mathrm{~m} \times 0.3 \mathrm{~m}$ rectangular geometry.

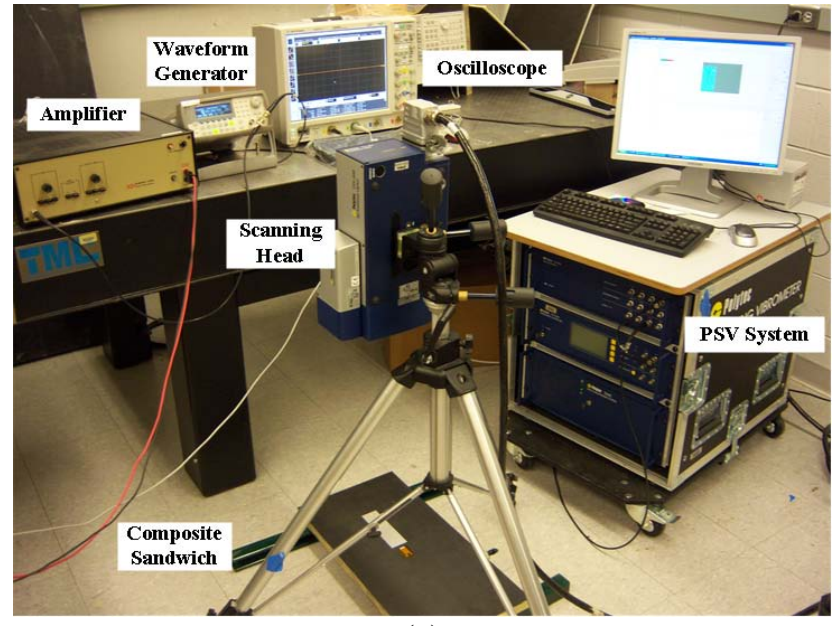

(a)

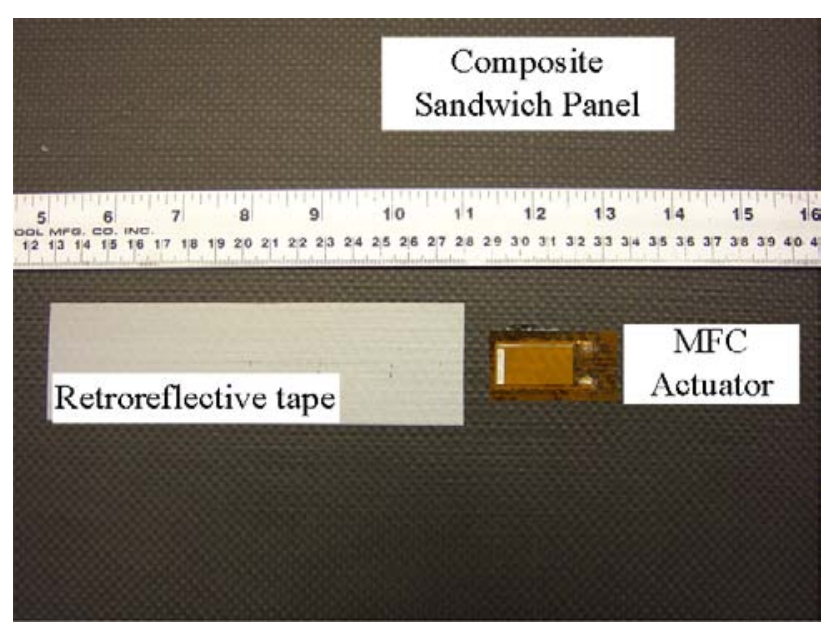

(b)

Figure 7. (a) Experimental setup showing different components used in the laser vibrometry experiments. (b) Composite sandwich specimen with MFC actuator and retro-reflective tape. 
Guided waves are generated in the composite sandwich specimen with the help of a macro fiber composite (MFC) actuator, as shown in Fig. 7(b), bonded onto the top surface of the panel. The actuator is oriented along the length of the panel and is placed at an optimum location to avoid boundary reflections. The transducer used in the experiments is 28-mm long and 15-mm wide, and it is excited with a 3.5-cycle Hann-modulated toneburst, shown in Fig. 3(b). GW were generated with center frequencies varying between $10 \mathrm{kHz}$ and $200 \mathrm{kHz}$ with an interval of 10 kHz. The GW field was measured using a Polytec PSV-400 scanning laser vibrometer by recording the out-of-plane velocities in a configuration as shown in Fig. 7(a). A patch of retro-reflective tape, shown in Fig. 7(b), is attached to the top and bottom surface to ensure consistent laser signal strength. The out-of-plane velocity is measured along a segment aligned with the actuator on the top surface and bottom surface of the composite sandwich panel. The amplitude of out-of-plane displacement calculated by integrating the velocity measured from the laser experiments is compared between the top surface and bottom surface.

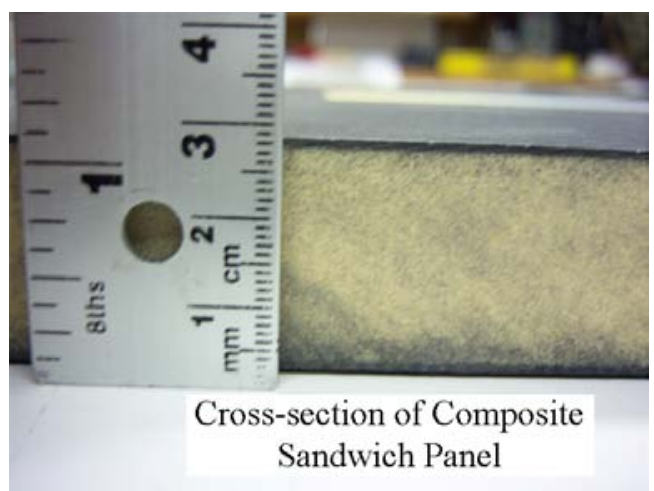

(a)

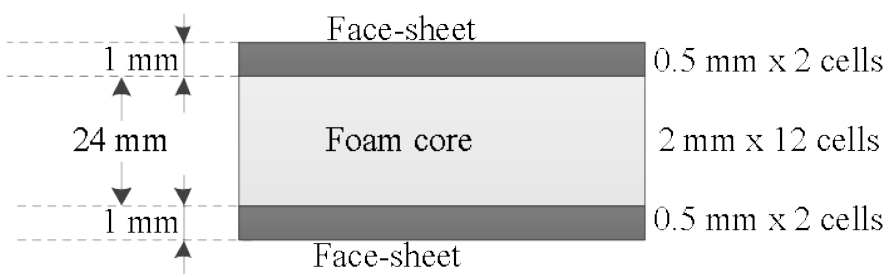

(b)

Figure 8. (a) Cross section of the composite foam core sandwich. (b) Cross-sectional schematic of the sandwich panel in LISA simulations.

Numerical simulations are performed on the composite sandwich panel with in-plane ( $X_{1} X_{2}$-plane) discretizations of $1 \mathrm{~mm} \times 1 \mathrm{~mm}$. The cross section of the sandwich panel (Fig. 8(a)) is discretized with facesheets having $\Delta x_{3}$ equal to $0.5 \mathrm{~mm}$ and foam core with $\Delta x_{3}$ equal to $2 \mathrm{~mm}$ ( Fig. 8(b)). The discretization was chosen to satisfy the CFL criterion and avoid excessive simulation times. As described in the experiments, the excitation is prescribed on the top surface of the model in the shape of a rectangular MFC. In-plane displacements along the length of the actuator parallel to $X_{1}$ were considered for actuation. The ouf-of-plane displacement was recorded on the top surface of the sandwich panel and Hilbert transform of the signal determined the peak of the arriving signal. The scaled ratio of amplitude of the signal on the bottom surface and top surface is plotted at different distances from the center of the actuator as shown in Fig. 9.

From Fig. 9 it is visible that lower frequencies are suitable for GW testing as more energy propagates to the bottom facesheet as compared to the higher frequencies, and the 3D numerical simulations based on LISA are able to capture the trend in the GW propagation characteristics accurately. More experiments and numerical simulations are underway at lower frequencies to investigate if the response at $10 \mathrm{kHz}$ is a global peak. 


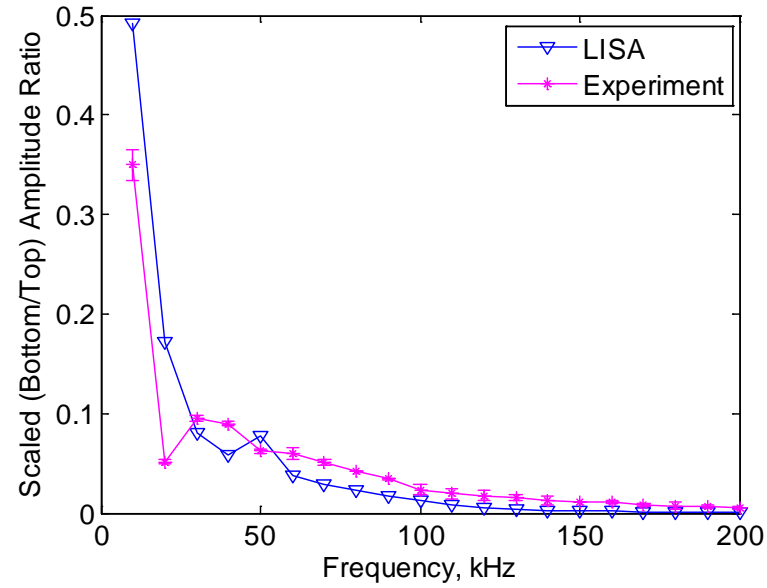

(a)

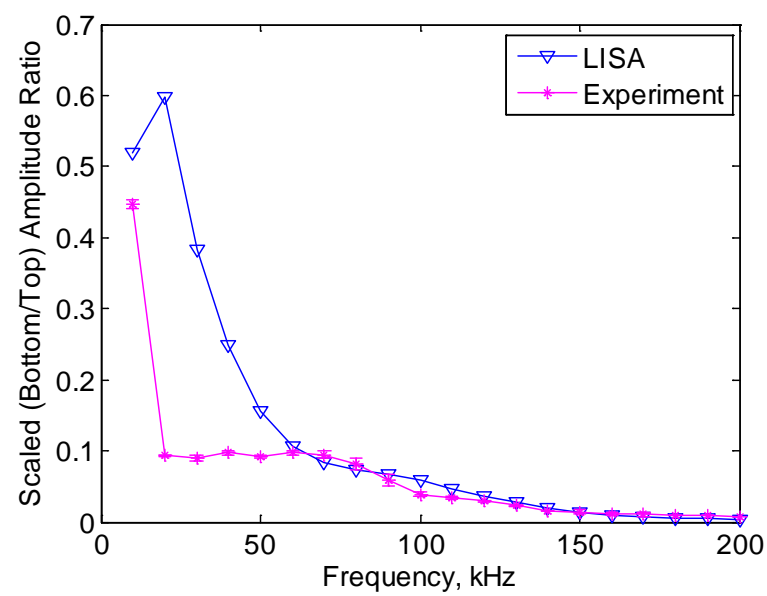

(c)

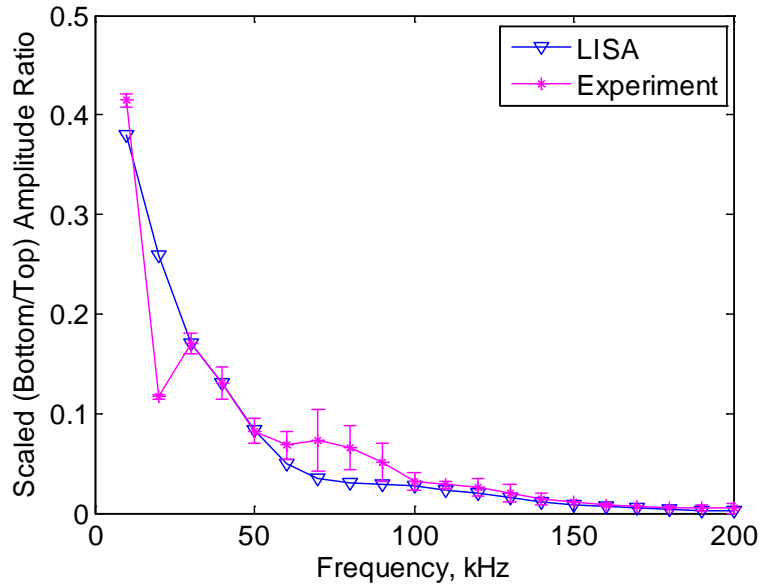

(b)

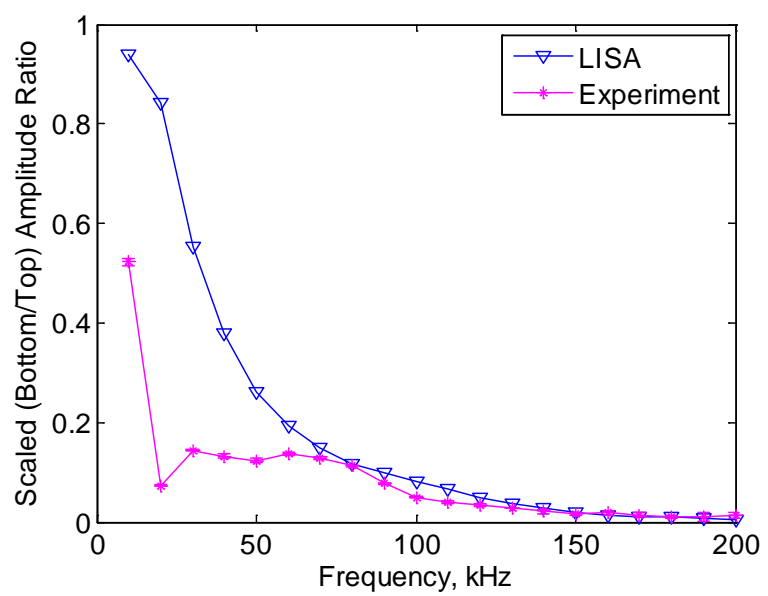

(d)

Figure 9. Ratio of the amplitudes of signals on the bottom surface and top surface for different frequencies at a distance of (a) $40 \mathrm{~mm}$, (b) $50 \mathrm{~mm}$, (c) $60 \mathrm{~mm}$ and (d) $70 \mathrm{~mm}$ from the center of the MFC actuator.

\section{Summary and Future Work}

Local interaction simulation approach (LISA) has been extended and implemented for a generalized orthotropic medium with non-uniform through-the-thickness discretization. Three-dimensional elastodynamic equilibrium equations were used in deriving the iterative equations with finite difference (FD) transformations, and the sharp interface method (SIM) was used to incorporate the heterogeneity from the sandwich construction. Studies with frequency variation were performed on an isotropic aluminum plate and uni-directional IM7 laminate and validated with DISPERSE. Numerical studies were also carried out on uni-directional IM7 laminate to verify the formulation for varying through-the-thickness discretizations. Finally, preliminary experimental and numerical studies were conducted on a composite foam core sandwich panel, and it was found that lower frequencies have a better potential for SHM-related applications. In the future, the modeling capability will be further validated in honeycomb core composite panels and more studies will be conducted on the existing foam core sandwich structures.

\section{Acknowledgments}

This work is supported by the NASA University Institutes Project/Lightweight Spacecraft, Structures, and Materials Program under grant Z634001. Claudia Meyer and Mark Shuart are the program managers, respectively. The authors thank Ken I. Salas (former PhD student in the research group, currently at GE Global Research) for the foam 
sandwich panel experimental results used in this paper. The authors would also like to thank Anthony M. Waas (Univ. of Michigan) for providing the composite sandwich specimen.

\section{References}

${ }^{1}$ Herrmann, J., Kim, J.-Y., Jacobs, L. J., Qu, J., Littles, J. W., and Savage, M. F., "Assessment of Material Damage in a Nickel-Base Superalloy Using Nonlinear Rayleigh Surface Waves," Journal of Applied Physics, Vol. 99, No. 12, 2006, p. 124913.

${ }^{2}$ Culshaw, B., Pierce, S.G., and Staszewski, W J., "Condition Monitoring in Composite Materials: An Integrated System Approach, " Proc. Inst. Mech. Eng. I, J. Syst. Control Eng. (UK), Vol. 212 , No. 13, 1998, pp.189-202.

${ }^{3}$ Raghavan, A., and Cesnik, C. E. S. "Review of Guided-Wave Structural Health Monitoring," The Shock and Vibration Digest, Vol. 39, No. 2, 2007, pp. 91-114.

${ }^{4}$ Lih, S.-S. and Mal, A. K., "On the Accuracy of Approximate Plate Theories for Wave Field Calculations in Composite Laminates," Wave Motion, Vol. 21, No. 1, 1995, pp. 17-34.

${ }^{5}$ Moulin, E., Assaad, J., and Delebarre, C., "Modeling of Lamb Waves Generated by Integrated Transducers in Composite Plates Using a Coupled Finite Element-Normal Mode Expansion," Journal of the Acoustical Society of America, Vol. 107, No. 1, 2000, pp. 87-94.

${ }^{6}$ Raghavan, A. and Cesnik, C. E. S., "Finite-dimensional Piezoelectric Transducer Modeling for Guided Wave Based Structural Health Monitoring," Smart Materials and Structures, Vol. 14, No. 6, 2005, pp. 1448-1461.

${ }^{7}$ Lowe, M. J. S., "Plate Waves for the NDT of Diffusion Bonded Titanium," Ph. D. Dissertation, Dept. of Mechanical Engineering, Imperial College of Science, Technology and Medicine, London, 1992.

${ }^{8}$ Sornette, D., Macon, L., and Coste, J., "Transfer-Matrix Theory of Leaky Guided-Waves," Journal De Physique, Vol. 49, No. 10, 1988, pp. 1683-1689.

${ }^{9}$ Strickwerda, J. C., Finite Difference Schemes and Partial Differential Equations Belmont: Wadsworth-Brooks, 1989.

${ }^{10}$ Zienkiewicz, O. C., The Finite Element Method,. 4th ed., McGraw-Hill, London, 1989.

${ }^{11}$ Brebbia, C. A., Tells, J. C. F., and Wrobel, L. C., Boundary Element Techniques: Theory and Applications in Engineering, Springer-Verlag, Berlin and New York, 1984.

${ }^{12}$ Delsanto, P. P., Schechter, R. S., and Mignogna, R. B., "Connection Machine Simulation of Ultrasonic Wave Propagation in Materials III: The Three-dimensional Case," Wave Motion, Vol. 26, No. 4, 1997, pp. 329-339.

${ }^{13}$ Sundararaman, S., and Adams, D. E., "Accuracy and Convergence Using a Local Interaction Simulation Approach in One, Two, and Three Dimensions," Journal of Applied Mechanics, Vol. 76, 2009, p. 031008.

${ }^{14}$ Sundararaman, S., and Adams, D. E., "Modeling Guided Waves for Damage Identification in Isotropic and Orthotropic Plates Using a Local Interaction Simulation Approach," Journal of Vibration and Acoustics, Vol. 130, No. 4, 2008 , p. 041009.

${ }^{15}$ Lee, B. C., and Staszewski, W. J., "Modelling of Lamb Waves for Damage Detection in Metallic Structures: Part I. Wave Propagation," Smart Materials and Structures, Vol. 12, No. 5, 2003, pp. 804-814.

${ }^{16}$ Lee, B. C., and Staszewski, W. J., "Modelling of Lamb Waves for Damage Detection in Metallic Structures: Part II. Wave Interactions With Damage," Smart Materials and Structures, Vol. 12, No. 5, 2003, pp. 815-824.

${ }^{17}$ Ruzzene, M., Jeong, S. M., Michaels, T. E., Michaels, J. E., and Mi, B., "Simulation and Measurement of Ultrasonic Waves in Elastic Plates Using Laser Vibrometry," AIP Conference Proceedings, Vol. 760, American Institute of Physics, Melville, New York, 2005, pp. 172-179.

${ }^{18}$ Agostini, V., Delsanto, P. P., Genesio, I., and Olivero, D., "Simulation of Lamb Wave Propagation for the Characterization of Complex Structures," IEEE Transactions on Ultrasonics, Ferroelectrics and Frequency Control Vol. 50, No. 4, 2003, pp. 441448.

${ }^{19}$ Agostini, V., Baboux, J.-C., Delsanto, P. P., Monnier, T., and Olivero, D., "Application of Lamb Waves for the Characterization of Composite Plates," AIP Conference Proceedings, Vol. 497, American Institute of Physics, Melville, New York, 1999, pp. 455-460.

${ }^{20}$ Nadella, K. S. and Cesnik, C. E. S., "Local Interaction Simulation of Guided-Wave Propagation in Composite Plates," Proceedings of SPIE, Vol. 7984, SPIE, Bellingham, Washington, 2011, p. 79841X.

${ }^{21}$ Sinor, M., "Numerical Modelling and Visualisation of Elastic Wave Propagation in Arbitrary Complex Media," Proceedings of the Eighth Workshop on Multimedia in Physics Teaching and Learning of the European Physical Society. KarlFranzens-Universität Graz, Austria, 2004.

${ }^{22}$ Alleyne, D. N., "The Nondestructive Testing of Plates Using Ultrasonic Lamb Waves," Ph.D. Dissertation, Dept. of Mechanical Engineering, Imperial College of Science, Technology and Medicine, London, 1991.

${ }^{23}$ Nadella, K. S. and Cesnik, C. E. S., " Numerical Simulation of Wave Propagation in Composite Plates," Proceedings of SPIE, Vol. 8348, SPIE, Bellingham, Washington, 2012.

${ }^{24} \mathrm{Ji}, \mathrm{W}$., "Static and Dynamic Response of a Sandwich Structure Under Axial Compression," Ph.D. Dissertation, Dept. of Aerospace Engineering, University of Michigan, Ann Arbor, Michigan, 2008.

${ }^{25}$ General Plastics Manufacturing Company, "FR-6710 product data sheet. Technical report,".[online database], URL: http://www.generalplastics.com/downloads/tds/fr-6710-tds.pdf [cited 30 March 2012].

${ }^{26}$ DISPERSE Software Package, Ver. 2.0.16i, Imperial College, United Kingdom, 2011. 